\title{
Echocardiography derived intra-ventricular pressure gradients: a window to the temporal and spatial components of diastolic dysfunction
}

\author{
Georgios K. Chalikias ${ }^{1}$ [D $\cdot$ Dimitrios N. Tziakas ${ }^{1}$
}

Received: 14 July 2021 / Accepted: 15 July 2021 / Published online: 20 July 2021

(c) The Author(s), under exclusive licence to Springer Nature B.V. 2021

Diastole begins in systole, as energy stored within the myocyte and as torsion in the interstitial fibers of the myocardium. As systole ends with aortic valve closure an abrupt untwisting occurs, which lowers pressure in the left ventricle (LV) (isovolumic relaxation) until the mitral valve opens, and blood flows along a negative pressure gradient (suction) toward the apex (rapid filling period) until the pressure equilibrates between the left atrium and the LV, resulting in diastasis until the final component of ventricular filling occurs with atrial contraction (Fig. 1) [1]. Derangement of any of these components may produce the pathophysiological entity of diastolic dysfunction [1].

In patients presenting with dyspnea or other symptoms of heart failure and normal LV ejection fraction on echocardiography, the diagnosis of diastolic dysfunction with preserved ejection fraction is not always straightforward [2]. This is especially challenging in chronic kidney disease (CKD) patients, in whom volume overload and falsely elevated N-terminal pro-brain natriuretic peptide (NT-proBNP) are common phenomena [3].

The hallmark of diastolic dysfunction is the impaired capacity to fill or maintain stroke volume without a compensatory increase in LV filling pressures [4]. Heart failure with preserved ejection fraction (HFpEF) patients typically have high LV filling pressures, whether at rest and/ or on exercise [5]. Therefore, it is reasonable to argue that the estimation of LV filling pressure is the more important aspect of evaluating LV diastolic function. Although various non-invasive echocardiographic measures exist to assess LV filling pressures such as mitral inflow Doppler velocities (E and A wave), tissue Doppler annular velocities ( $\mathrm{e}^{\prime}$ wave),

Georgios K. Chalikias

gchaliki@med.duth.gr

1 Cardiology Department, Medical School, Democritus University of Thrace, Dragana, 68100 Alexandroupolis, Greece tricuspid regurgitation velocity, E/e' ratio and left atrial volume, their clinical performance has been characterized at best as "good" or "reliable" [6, 7]. In addition, the fact that the various parameters used are subject to fundamental limitations and reflect different physiological aspects of diastole has led to substantial ambiguity [7]. More importantly, the dynamic nature of LV filling pattern in a three-dimension plane, the formation of vortices by blood flow as well as the spatial-temporal distribution of the velocity of blood flow from the annulus to the apex, suggests that any accurate estimation of LV filling pressure, includes the development and validation of other indices of $\mathrm{LV}$ diastolic function regional in nature given the limitations of the existing methodologies $[8,9]$.

Regional intra-cavity pressure differences between various parts of the LV have been recognized for some time, however the potential clinical importance of these intraventricular pressure gradients (IVPG) within the LV during diastole and systole have only more recently gained attention [9]. These diastolic IVPGs may play an important role in ventricular filling and emptying in the normal heart [9]. Initially, Courtois et al. observed, in a canine model, a significant early diastolic pressure gradient along the LV inflow tract with minimum pressure in the apex suggesting suction of the blood toward the LV apex $[9,10]$. Later, Smiseth et al. demonstrated, in humans, the presence of a diastolic IVPG between the apex and outflow tract. They showed that when apical pressure started to rise, outflow pressure continued to fall, thus creating an apex-to-outflow tract pressure gradient in early diastole $[9,11]$. In late diastole, during atrial contraction, there was also a gradient from the apex-to-outflow tract. Furthermore, regional pressure-wave pattern recorded during atrial contraction is exactly opposite to the pattern recorded during the early rapid-filling phase [9, 12]. Finally, Iwano et al. showed that basilar IVPG is mainly affected by LA pressure [13]. Based on these complex spatial and temporal physiological IVPGs observed during ventricular 
Fig. 1 Left ventricular, left atrial and aortic pressure curves during cardiac cycle. Phases of LV diastole are isovolumic relaxation, rapid filling period, diastasis and atrial contraction period. Adapted from Daniel Chang, MD (revised original work of Destiny Qx; Redrawn as SVG by xavax) with permission Creative Commons Attribution-Share Alike 2.5 Generic

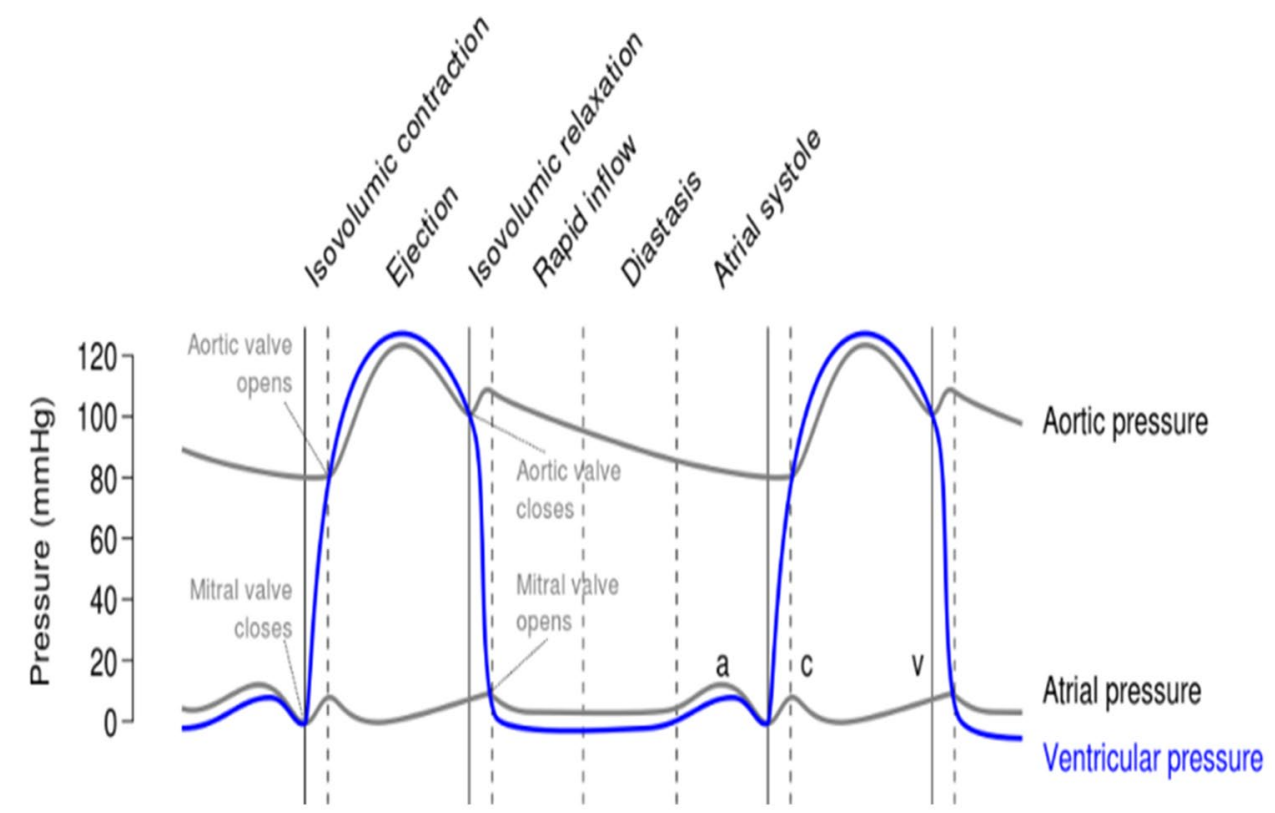

filling can be inferred that filling is completed first in the apex and only then in the base and in the LV outflow tract thus preparing for an efficient ventricular emptying (Fig. 2) [9]. Therefore, IVPGs are probably more reliable markers for ventricular relaxation, elastic recoil, diastolic suction and efficient LV filling [9].

In previously reported studies, most IVPG data were measured during diastole by color M-mode Doppler (CMMD) echocardiographic imaging. Greenberg et al. used the local spatial and temporal velocity distribution measured by color Doppler M-mode echocardiography to calculate local pressure gradients using the Euler equation, integration of which allows them to calculate a pressure difference between two points along the inflow tract
[14]. Accuracy of the method depends on the temporal and spatial resolutions of color Doppler M-mode images and is also related to the degree to which the ultrasound scanline approximates an inflow streamline through the center of the mitral valve [15]. Moreover, this one-dimensional measurement has been challenged by the multidimensional velocities of the intra-ventricular flow [15]. Finally, this method is limited because the transmitral velocity profile is also affected by several parameters other than LV diastolic function, such as heart rate, atrioventricular conduction interval and left atrial pressure [9]. As a reliable non-invasive method to characterize the state of LV relaxation is still an unsolved issue additional techniques are researched such as incorporation of tissue Doppler
Fig. 2 Spatial and temporal profile of intra-cavity pressures in healthy left cardiac chambers. Adapted from Pedrizzetti G, La Canna G, Alfieri O, Tonti G. The vortex-an early predictor of cardiovascular outcome? Nat Rev Cardiol. 2014 Sep;11(9):545-53

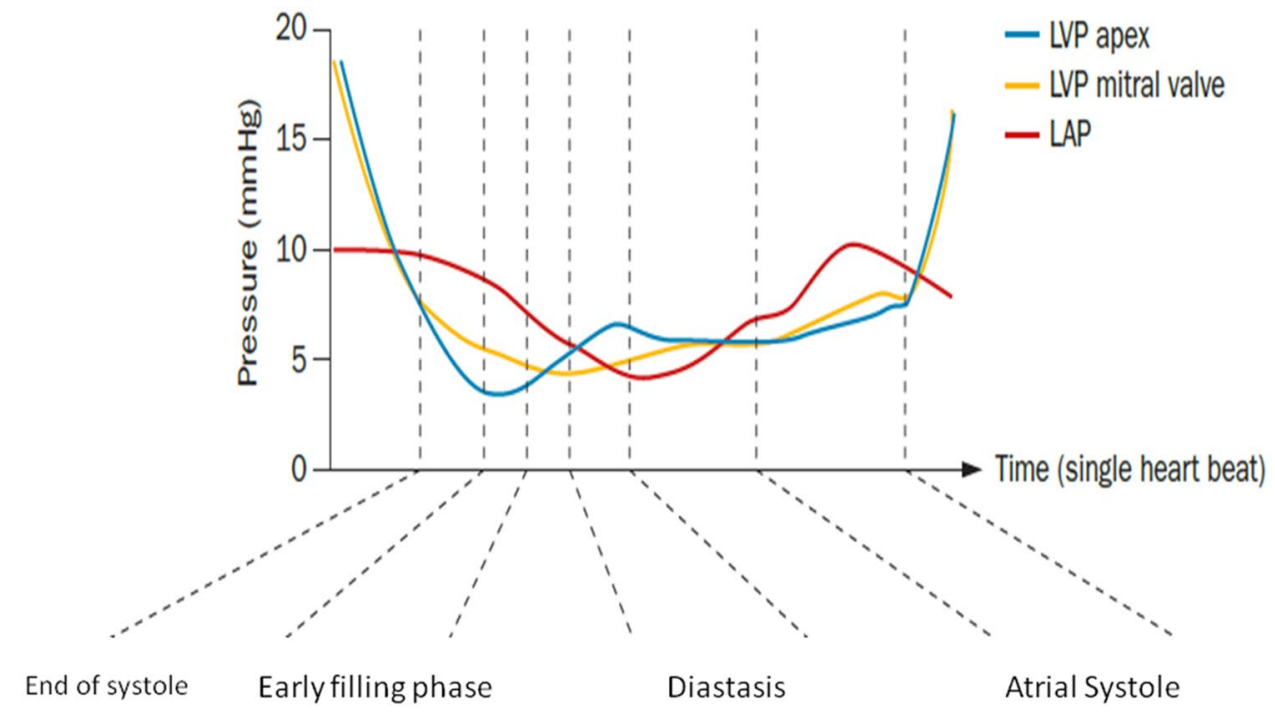


imaging [16] or strain mapping [17], vector flow mapping (VFM) [18], contrast echocardiography-particle image velocimetry (CE-PIV) [8], and relative pressure imaging (RPI) in left ventricle using VMF (RPI-VMF) [19]. Flow-derived IVPG parametric maps offer an opportunity to improve methods for assessing LV filling as they are based on two-dimensional (2D)-VFM velocity fields and are angle-independent, however interpretation of their results should be made with caution [8].

In the present issue of the International Journal of Cardiovascular Imaging, Zhong et al. [20] assessed differences and associations between early diastolic IVPGs using relative pressure imaging derived from VFM in 51 patients with CKD (32 with HFpEF and 19 without) and 39 healthy controls. Significant differences were present in mid- and apical early diastolic IVPG between CKD patients with HFpEF and those without as well as between CKD HFpEF patients and controls. Finally, a reduced apical IVPG was associated with a composite end-point including all cause death and cardiovascular hospitalizations in the CKD population. This interesting study is among the first to assess IVPGs as markers for diastolic dysfunction. Although, IVPGs may be a useful tool in diagnosing HFpEF in CKD patients, several limitations should be taken into account. Firstly, the authors did not compare IVPG neither with other echocardiographic indices such as CMMD nor with data derived from cardiac catheterization. More alarmingly, early diastolic IVPGs were weakly correlated with standard echocardiographic indices of HFpEF. Although observation raise a certain amount of concern, it is logical to postulate that early diastolic IVPGs could represent a more sensitive marker capable of detecting diastolic dysfunction earlier compared to the standard echocardiography markers. Furthermore, cardiac pre-load was not assessed methodologically in CKD patients, a patient population in whom volume status is susceptible to variation due to therapeutic interventions. Finally, the prognostic ability of early diastolic IVPG is subject to inherent limitations of the relative analysis due to the small size of the study population.

Nevertheless, echocardiography derived IVPGs estimation emerges as a novel, useful and potentially clinically applicable mean to identify diastolic abnormalities earlier in their course and more comprehensively in their temporal and spatial properties. Future, larger in size and more elaborate in methodology, studies would certainly delineate further the impact of IVPG echocardiography assessment as an additional clinical tool for the diagnosis of diastolic dysfunction.

Funding None.

\section{Declarations}

Conflict of interest The authors have no conflict of interests to disclose.

Ethical approval This editorial comment is compliant with ethical standards. Ethics approval is not applicable to this manuscript.

\section{References}

1. Mitter SS, Shah SJ, Thomas JD (2017) A test in context: E/A and $\mathrm{E} / \mathrm{e}^{\prime}$ to assess diastolic dysfunction and LV filling pressure. J Am Coll Cardiol 69(11):1451-1464

2. Ponikowski P, Voors AA, Anker SD et al (2016) ESC Scientific Document Group. 2016 ESC Guidelines for the diagnosis and treatment of acute and chronic heart failure: the Task Force for the diagnosis and treatment of acute and chronic heart failure of the European Society of Cardiology (ESC) Developed with the special contribution of the Heart Failure Association (HFA) of the ESC. Eur Heart J 37(27):2129-2200

3. Ogawa T, Nitta K (2018) Clinical impact of left ventricular diastolic dysfunction in chronic kidney disease. Contrib Nephrol 195:81-91

4. Leite-Moreira AF (2006) Current perspectives in diastolic dysfunction and diastolic heart failure. Heart 92:712-718

5. Pieske B, Tschöpe C, de Boer RA et al (2019) How to diagnose heart failure with preserved ejection fraction: the HFA-PEFF diagnostic algorithm: a consensus recommendation from the Heart Failure Association (HFA) of the European Society of Cardiology (ESC). Eur Heart J 40(40):3297-3317

6. Sato K, Grant ADM, Negishi K et al (2017) Reliability of updated left ventricular diastolic function recommendations in predicting elevated left ventricular filling pressure and prognosis. Am Heart J 189:28-39

7. Lancellotti P, Galderisi M, Edvardsen T et al (2017) Echo-Doppler estimation of left ventricular filling pressure: results of the multicentre EACVI Euro-Filling study. Eur Heart J Cardiovasc Imaging 18(9):961-968

8. Pedrizzetti G, La Canna G, Alfieri O, Tonti G (2014) The vortexan early predictor of cardiovascular outcome? Nat Rev Cardiol 11(9):545-553

9. Guerra M, Sampaio F, Brás-Silva C, Leite-Moreira AF et al (2011) Left intraventricular diastolic and systolic pressure gradients. Exp Biol Med (Maywood) 236(12):1364-1372

10. Courtois M, Kovács SJ, Ludbrook PA (1988) Transmitral pressure-flow velocity relation. Importance of regional pressure gradients in the left ventricle during diastole. Circulation 78(3):661-671

11. Smiseth OA, Steine K, Sandbaek G, Stugaard M, Gjolberg T (1998) Mechanics of intraventricular filling: study of LV early diastolic pressure gradients and flow velocities. Am J Physiol 275(3):H1062-H1069

12. Courtois M, Kovács SJ, Ludbrook PA (1990) Physiological early diastolic intraventricular pressure gradient is lost during acute myocardial ischemia. Circulation 81(5):1688-1696

13. Wano H, Kamimura D, Fox E, Hall M, Vlachos P, Little WC (2015) Altered spatial distribution of the diastolic left ventricular pressure difference in heart failure. J Am Soc Echocardiogr 28(5):597-605

14. Greenberg NL, Vandervoort PM, Firstenberg MS, Garcia MJ, Thomas JD (2001) Estimation of diastolic intraventricular pressure gradients by Doppler M-mode echocardiography. Am J Physiol Heart Circ Physiol 280:2507-2515 
15. Londono-Hoyos FJ, Swillens A, Van Cauwenberge J et al (2018) Assessment of methodologies to calculate intraventricular pressure differences in computational models and patients. Med Biol Eng Comput 56(3):469-481

16. Yotti R, Bermejo J, Benito Y et al (2011) Noninvasive estimation of the rate of relaxation by the analysis of intraventricular pressure gradients. Circ Cardiovasc Imaging 4(2):94-104

17. Kylmälä MM, Antila MK, Kivistö SM et al (2008) Tissue Doppler strain-mapping in the assessment of the extent of chronic myocardial infarction: validation using magnetic resonance imaging. Eur J Echocardiogr 9(5):678-684

18. Berlot B, Moya Mur JL, Jug B et al (2019) Effect of diastolic dysfunction on intraventricular velocity behavior in early diastole by flow mapping. Int J Cardiovasc Imaging 35(9):1627-1636
19. Tanaka T, Okada T, Nishiyama T, Seki Y (2017) Relative pressure imaging in left ventricle using ultrasonic vector flow mapping. Jpn J Appl Phys 56(7S1):07JF26

20. Zhong Y, Cai Y, Liu M, Bai W, Wang F, Tang H, Rao L. (2021) Left ventricular diastolic pressure gradient and outcome in advanced chronic kidney disease patients with preserved ejection fraction. Int J Cardiovasc Imaging

Publisher's Note Springer Nature remains neutral with regard to jurisdictional claims in published maps and institutional affiliations. 